\title{
The corneomandibular reflex ${ }^{1}$
}

\author{
ROBERT M. GORDON ${ }^{2}$ AND MORRIS B. BENDER
}

From the Department of Neurology, the Mount Sinai Hospital, New York, U.S.A.

SUMMARY Seven patients are presented in whom a prominent corneomandibular reflex was observed. These patients all had severe cerebral and/or brain-stem disease with altered states of consciousness. Two additional patients with less prominent and inconstant corneomandibula reflexes were seen; one had bulbar amyotrophic lateral sclerosis and one had no evidence of brain disease. The corneomandibular reflex, when found to be prominent, reflects an exaggeration of the् normal. Therefore one may consider the corneomandibular hyper-reflexia as possibly due to diseasew of the corticobulbar system.

The corneomandibular reflex consists of an involuntary contralateral deviation and protrusion of the lower jaw during corneal stimulation. It is not a common phenomenon and has been rediscovered several times since its initial description by Von Sölder in 1902. It is found mostly in patients with brain-stem or bilateral cerebral lesions who are in coma or semicomatose.

There have been differing opinions as to the incidence, anatomical basis, and clinical significance of this reflex. Assessment of the literature on the subject has been made difficult because of the paucity of clinical descriptions of patients who have demonstrated this phenomenon. We have noticed certain characteristics common to the patients in whom we have elicited corneomandibular reflexes. These observations form the basis of this communication.

\section{PATIENTS}

In the course of routine clinical examinations of neurological patients at The Mount Sinai Hospital, New York, and the U.S. Public Health Service Hospital, Norfolk, Virginia, from January 1966 to June 1969, eight patients with corneomandibular reflexes were encountered. No special attempt was made to elicit the reflex in these patients. Rather, the phenomenon was encountered by chance.

Of the eight patients in the series, one had only a

\footnotetext{
1This paper was presented in part before a meeting of the Houston Neurological Society on 10 September 1969. It was supported by Research Grants NB 05221 and NB 05072.

${ }^{2}$ Reprint address: Department of Neurology, Mt. Sinai School of Medicine, 100th Street and Fifth Avenue, New York, N.Y. 10029, U.S.A.
}

weak bilateral response on a few occasions. This was a woman with bulbar and spinal amyotrophic lateral sclerosis. The other seven patients had prominent and consistently elicited cornes $\omega$ mandibular reflexes. The clinical features common तुow these patients were (1) the presence of bilateg corneomandibular reflexes, in some cases m\%e prominent on one side; (2) a depressed state of cenf $\vec{c}$ sciousness, usually coma; and (3) the presence 815 severe neurological abnormalities, usually moter, $\stackrel{\oplus}{\oplus}$ in addition to the depressed state of consciousness. $\overrightarrow{0}$ Four patients died and three of these had post mortem examinations of the nervous system.

\section{CASE 1}

This 61 year old man was admitted to the U.S. Public气्ญ Health Service Hospital, Norfolk, Virginia on 16 Feb_ ruary 1966 after a generalized seizure. For approximately $\overrightarrow{\vec{O}}$ two weeks before this he had complained of headache, 3 photophobia, and anorexia. In the three day period immediately preceding admission increasing lethargy hado been noted. On admission to the hospital he had several more generalized seizures, most of which began with a tonic deviation of the head and eyes to the right.

On examination the patient was deeply stuporous, grunting, at times, in response to commands and painful? stimuli. His head and eyes were generally turned to the left, but with cephalic manoeuvring the eyes could be 3 moved to the right. The pupils were $2 \mathrm{~mm}$ in diameter,o equal and reactive to light. He responded to painful stimuli and moved all four limbs equally. The deep tendon reflexes were depressed. The plantar responses were flexor bilaterally. The neck was supple.

Firm stimulation of either cornea with a sterile cottonapplicator resulted in simultaneous closure of both eyes, $N$ and, in addition, a strong unilateral deviation of the jawN to the side opposite the stimulated cornea. At the same 
time the patient's mouth opened slightly (Fig.). The jaw then returned to its resting position within $1 \frac{1}{2}$ seconds. This patterned response could be elicited three or four times in rapid succession. Subsequently, there was fatigue, but after waiting several seconds it could again be elicited. In this patient the response could also be produced with a very light corneal stimulation, such as a lightly applied wisp of cotton, but the corneomandibular response was then less prominent. Stimulation of other areas of the face by touch, pinprick, and pressure over the eyelids did not produce this phenomenon. Passive movements of the jaw failed to produce eyelid or eyeball movements.

A spinal tap revealed bloody cerebrospinal fluid with a xanthochromic supernatant under a pressure of $70 \mathrm{~mm}$ of water. A left carotid arteriogram was normal.

The next day the patient became more stuporous and lapsed into a deep coma with dilated pupils, divergent eyes, and absent ocular deviation on cephalic turning. In response to painful stimuli there were pronation and extension movements of the arms and extensor movement of the legs. During the comatose state the corneomandibular reflex was still easily elicited bilaterally. The patient died on 17 February 1966.

At post mortem examination of the brain a recent thrombus was found in the left middle cerebral artery. There was haemorrhagic infarction of the left frontal, parietal, and occipital lobes, swelling of the left cerebral hemisphere, and herniation of the uncus of the left temporal lobe through the tentorial notch. Haemorrhages were seen within the tegmentum of the pons.

In summary, this patient with bilateral corneomandibular reflexes showed clinical evidence of a left cerebral hemisphere lesion in the presence of a diffuse disturbance of cerebral and brain-stem function. This was confirmed at necropsy, which showed a massive haemorrhagic infarction of the left cerebral hemisphere with uncal herniation and secondary brain-stem haemorrhages.

\section{CASE 2}

This 60 year old man had an episode of left leg weakness lasting a few minutes in February 1966. A few days after this he noticed weakness of his right arm, which persisted for three days and then cleared completely. On 17 March 1966, his left leg became weak, temporarily improved, and then on 24 March 1966, became completely useless. He became progressively stuporous over the next six days. There was a past history of diabetes mellitus, hypertension, and an old myocardial infarction.

Neurological examination revealed him to be unreactive to all but deeply painful stimuli, in response to which he feebly moved his right side extremities. There were Cheyne-Stokes respirations. Examination of the optic fundi revealed numerous varying sized haemorrhages, exudates, and microaneurysms. The optic discs were flat. There were random conjugate movements of the eyes in both directions. The pupils were normal in size, equal, and reactive to light. The deep tendon reflexes were active, somewhat more so on the left. There was a left Babinski sign. The right plantar reflex was equivocal.

Firm stimulation of either cornea with a sterile applicator was followed by a bilateral blink response accompanied by a marked deviation and some protrusion of the jaw to the side opposite the stimulated cornea lasting one to two seconds. This response could be elicited on repeated testing, but only by firm stimulation of the cornea, and not by stimulation of the sclera, face, body, or by ice water caloric irrigation of either eardrum (which did produce ipsilateral deviation of the eyes conjugately

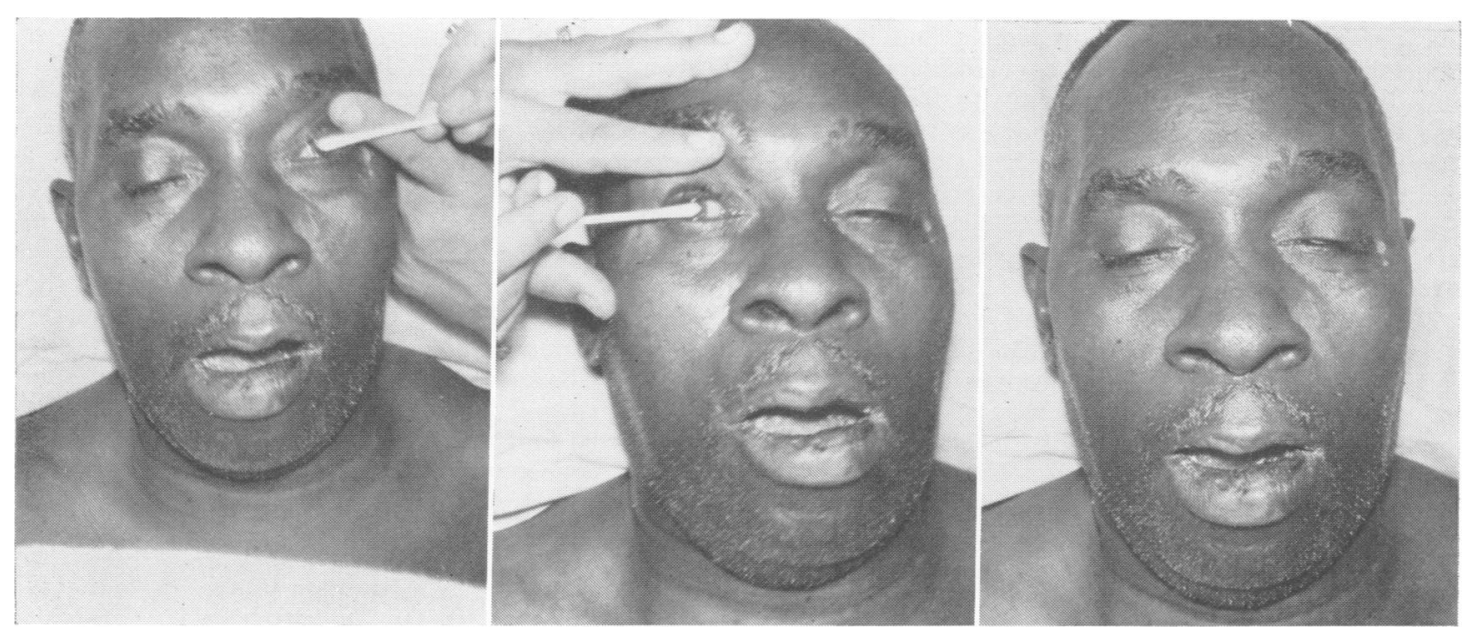

FIGURE. Case 1, illustrating the corneomandibular reflex. Right: with no corneal stimulation the jaw is midline. Centre: with right corneal stimulation the jaw deviates to the left. Left: with left corneal stimulation the jaw deviates to the right. 
without nystagmus). Flashing a bright light into either eye caused a bilateral blink response, but no deviation of the jaw.

Radiographs of the skull and the results of a lumbar puncture were normal. A right carotid arteriogram performed on 30 March 1966, demonstrated a complete occlusion of the right internal carotid artery at its origin. The patient remained in coma and died on 13 May 1966.

Post mortem examination of the head revealed a recent thrombus completely occluding the right internal carotid artery. There was marked atherosclerotic narrowing of the left internal carotid artery at its origin. The brain showed encephalomalacia of the right temporoparietal and left fronto-temporal hemispheres extending to the basal ganglia on both sides. No abnormalities were noted in the brain-stem or cerebellum.

In summary, this patient, demonstrating strong bilateral corneomandibular reflexes, had clinical eviderice of bilateral brain lesions. This $x$ as confirmed at necropsy, which showed bilateral extensive encephalomalacia of the cerebral hemispheres to the basal ganglia.

\section{CASE 3}

The patient, a 62 year old man, had complained for four years of severe headaches and episodes of flashing lights seen on his right side. In September 1967 he noticed a very severe headache, some difficulty with his speech, and trouble with his vision on the right side. He improved and did well until 24 April 1968, when he awakened from a nap with weakness of his right leg and face. He became confused, had difficulty with his speech, and complained of a severe headache.

Neurological examination revealed him to be alert. His speech was sparse, consisting of only one or two word responses, at times appropriate and at times consisting of nonsense words and syllables in a jargon type of speech. The patient was able to follow simple, but not complicated instructions. At times he would inappropriately burst into tears. There was an inability to deviate the eyes past the midline to the right. There was a right spastic hemiplegia with weakness of the right side of the face. The deep tendon reflexes were hyperactive bilaterally, more so on the right, with sustained right and transient left ankle clonus. The plantar reflexes were frequently extensor bilaterally.

Touching the right cornea firmly with a sterile applicator produced a closing motion of the patient's halfopen mouth with a deviation of his jaw to the left. This was immediate and lasted less than one second. The strength of the reflex and the ability to elicit it varied from time to time. In addition the reflex could not be elicited more than two or three times in rapid succession, but if one waited 15 seconds and tried again it could be elicited. A firm touch applied to the left cornea produced a deviation of the jaw to the right. However, this was elicited less frequently and seemed to 'tire' more quickly.

Pertinent laboratory studies were blood tinged xanthochromic spinal fluid under a pressure of $300 \mathrm{~mm}$ water, and an abnormal electroencephalogram which contained irregular 1.2 to $3 \mathrm{~Hz}$ slowing, left more than right. $A \bar{z}$ left carotid arteriogram demonstrated an avascular high $\mathbb{C}$ convexity frontoparietal mass.

The patient improved and was discharged on 9 June 1968. His condition remained stable until 14 April 1969 , when he suddenly became comatose. He died two days 0 later. Unfortunately, he was not seen by us during his terminal hospitalization.

A post mortem examination of the brain revealed a recent large frontal lobe haemorrhage with rupture into the lateral ventricle. In addition, there was a sharply circumscribed area of cavitation with yellowish discolouration in the left parietal lobe. This was associatedwith an old focal haemorrhage of the central white $\frac{C}{0}$ matter which had ruptured into the left lateral ventricle. $\frac{\overline{0}}{\overline{0}}$ The brain-stem and cerebellum were normal.

In summary, this patient with bilateral asymmetrical corneomandibular reflexes had clinical $\vec{\circ}$ evidence of a left intracerebral haemorrhage with bilateral brain dysfunction at the time he was $\vec{\omega}$ examined. He later developed an intracerebral haemorrhage on the right side. Post mortem examination showed that his initial left cerebral haemor- $\omega$ rhage had ruptured into the ventricular system + Thus, there was evidence for a diffuse disturbange i of brain function at the time of the initial exarp ination.

CASE 4

A 41 year old man underwent the removal of a 100 (1) sphenoidal ridge meningioma in 1962. In January 1968 $\vec{a} \overrightarrow{0}$ right parasagittal meningioma was removed. In Mare 1968 he complained of progressive unsteadiness in his left . sided extremities and indistinctness of his speech. He was hospitalized. A right brachial arteriogram was suggestive of a mass lesion in the left cerebellopontine angle. To confirm this a transfemoral aortic arch angiogram was performed on 27 March 1968, and after this procedure $\frac{}{D}$ the patient lapsed into coma and remained in this state. $\varrho$

On examination, while he was in coma, he was un- $\overrightarrow{\overrightarrow{0}}$ responsive to all but deeply painful stimuli to which he 3 responded by elevation and flexion of his left arm. His only spontaneous movements were coughing, irregular bilateral blinking, and periodic, fairly regular, approximately 3 per second extensor movements of his right wrist and ankle. His eyes deviated to the left with slow random conjugate movements in his left eyefield. Oculo- $\frac{5}{3}$ cephalic reflexes were diminished in the horizontal and vertical planes. The pupils were $4 \mathrm{~mm}$ in diameter, equal, $\stackrel{\circ}{2}$ and reacted to light. Extensor tonus was increased in his $₹$ limbs. The deep tendon reflexes were hyperactive. There 을 was a left Babinski sign. Suck, snout, and right palmomental reflexes were present.

On vigorous touching of the right cornea there was a bilateral blink response. Simultaneously, the lower jaw $N$ protruded and deviated to the left. This latter response $N$ was inconstant. On touching the left cornea there was a $O$ bilateral blink response and simultaneously the jaw pro- $\mathcal{\omega}$ truded and deviated to the right. This response was 
constant. No movement of the jaw was noted during spontaneous blinking.

The patient remained in coma. The corneomandibular reflexes as described were found whenever their elicitation was attempted. The patient died 29 April 1969. Necropsy was not performed.

In summary, this patient with clinical evidence of cerebral and probably brain-stem damage demonstrated bilateral, asymmetrical corneomandibular reflexes.

\section{CASE 5}

This 32 year old hypertensive woman underwent surgery in order to correct a stenosis of her right renal artery on 4 April 1966. Postoperatively she was stuporous and was placed upon a hypothermia blanket. She became more alert over the next two days, then again gradually lapsed into coma by 8 April 1966 .

On examination she appeared quite restless with her eyes open, apparently looking about. She was aphonic except for an occasional shrill cry: the optic fundi disclosed bilateral haemorrhages around the optic discs. Her eyes moved spontaneously and conjugately in the horizontal plane. Her pupils were equal and reacted to light. Her mouth was usually held closed and rigid, except when she opened it to cry out. There was no response to pinprick stimulation over her body. The deep tendon reflexes were hyperactive including the jaw jerk. The plantar reflex was neutral. A snout reflex was present.

Stimulation of either cornea produced bilateral eyelid closure, a wrinkling of the chin bilaterally, and a forceful contralateral deviation of the jaw lasting over one second. This could be produced only by firmly touching the cornea. A lesser degree of corneal stimulation produced wrinkling of the chin, but no contralateral movement of the mandible.

A spinal puncture on 14 April 1966 revealed an opening pressure of $290 \mathrm{~mm}$ of water. The fluid was otherwise normal. A skull series and bilateral carotid arteriograms were normal.

The patient remained in this coma-like state. She developed periodic fluctuation in muscle tonus, numerous adventitious movements of the jaw and extremities, and spontaneous horizontal nystagmoid movements of her eyes. At times she demonstrated predominantly flexor and at other times predominantly extensor rigidity. The corneomandibular reflexes persisted.

In summary, this patient with active bilateral corneomandibular reflexes had clinical evidence of severe bilateral cerebral and brain-stem damage.

\section{CASE 6}

A 20 year old female college student developed numbness of the extremities in December 1967. With this, she became progressively weak, developed neck pain, nausea, vomiting, headache, double vision, and drowsiness. There was increasing somnolence and weakness of all her extremities. She was then admitted to the Mount Sinai Hospital.

A general physical examination was normal. Neurological examination revealed responsiveness only to painful stimuli by grunting and grimacing. The optic discs were pale. The right pupil was $4 \mathrm{~mm}$, and the left $3 \mathrm{~mm}$ in diameter. Neither reacted to light. The eyes tended to be divergent and moved disconjugately in the horizontal plane. There was nystagmus in either eye alone or together, varying from time to time. There were no adduction movements during spontaneous horizontal ocular deviations or those induced by oculocephalic manoeuvres. There were frequent champing and grinding movements of the jaw. There were no spontaneous movements of the limbs, but the left leg moved in response to plantar stimulation. The deep tendon reflexes were increased in the upper and absent in the lower extremities.

Stimulation of either cornea produced a bilateral blink response; simultaneously the jaw deviated contralaterally (corneomandibular reflex), returning to its resting position in about one second. Concurrently there was a wrinkling of the ipsilateral chin. The corneomandibular response could be elicited only by firm stimulation of the cornea. Touching the sclera produced a bilateral blink response, but no jaw deviation. Pinprick, vibratory and tactile stimuli, over the eyeball, face or body, as well as ice water caloric irrigation of the eardrums produced no jaw deviation.

The patient remained in the hospital for one year during which time she underwent numerous diagnostic tests, none of which elucidated the nature of her illness. During this time, she consistently demonstrated the corneomandibular reflex. In addition she had, at various times, grasp, avoidance, sucking, snout, palmomental, Babinski, and tonic foot reflexes. She showed gradual, but slight improvement in her state of consciousness and ability to move her extremities. At the time of her discharge on 8 February 1969, there was still severe neurological impairment including bilateral corneomandibular reflex.

In summary, this patient with an obscure, but obviously diffuse disease of the nervous system showed bilateral corneomandibular reflexes, which persisted during the entire observation period of one year.

\section{CASE 7}

On 9 November 1968 this 58 year old man noted sudden inability to express himself lasting approximately two minutes. He had a second such episode two hours later, and a third episode on 13 November 1968. Early in December 1968 he was found on the floor with right side weakness. This progressed, and he became unable to express himself over the next several days. In July 1968 there had been an episode of transient weakness of his left hand.

On examination he was virtually aphonic except for an occasional grunt. He appeared to be aware of his environment, but gave little evidence of understanding anything. There was a severe right hemiparesis and hemisensory 
defect to pinprick stimulation including the face. At times both plantar responses were extensor, the right more consistently than the left. There were bilateral grasp reflexes, but no sucking or snout reflexes.

There was strong deviation of the jaw to the left when the right cornea was stimulated, and a less distinct and more rapid deviation of the jaw to the right when the left cornea was stimulated. This left corneomandibular reflex was not always present.

Pertinent laboratory data included a spinal fluid containing a protein of $74 \mathrm{mg} / 100 \mathrm{ml}$. An electroencephalogram was interpreted as showing left-sided abnormality in the presence of bilateral cerebral dysfunction. A left carotid arteriogram demonstrated a large suprasylvian vascular mass with arteriovenous shunting which was interpreted as a malignant brain tumour.

With corticosteroid and radiation treatment there was some improvement over the next five weeks. On 28 February 1969 there appeared the first of several focal right-sided seizures. He became progressively worse, and as of August 1969 he remained in a very lethargic state. The patient ultimately died in November 1969 and there was no necropsy.

In summary, this patient with a left cerebral neoplasm and evidence of bilateral cerebral dysfunction, demonstrated bilateral corneomandibular reflexes, the most prominent being contralateral to his major lesion.

\section{INCIDENCE OF CORNEOMANDIBULAR REFLEX}

In order to determine the incidence of the corneomandibular reflex in a random series of other neurological cases and to define more precisely the patient population in which it occurs, 300 randomly selected patients were examined specifically in an attempt to elicit the corneomandibular reflex. These patients were observed on the neurology services of the Mount Sinai and Elmhurst General Hospital, and represented the spectrum of neurological illness seen at these hospitals. Elicitation of the corneomandibular reflex was attempted in the same manner as already described. Only one instance of a corneomandibular reflex was observed. In this case the reflex was weak, transient, and bilateral. The patient in whom this occurred was a 42 year old woman with a thoracic myelopathy due to lymphosarcoma. There was no evidence of brain disease in this patient, and we have no follow up.

It was incidentally observed that a number of these 300 patients, many of whom had neurological disorders not involving the brain, demonstrated an ipsilateral or bilateral wrinkling of the chin, apparently due to contraction of the mentalis and perhaps other muscles in this area. This response was not associated with other movements of the jaw. We did not attempt to otserve the precise incidence of this corneomental reflex, as it may be termed, but it occurred in approximately 10 to $15 \%$ of our subjects regardless of neurological status, and in a few patients with no known brain disease.

\section{DISCUSSION}

In 1902, von Sölder first described the corneomandibular reflex, and for the next two decades he tenaciously defended the priority of his discovery in the German literature (von Sölder, 1902, 1918). He felt that the phenomenon was 'a physiological reflex, which, in certain pathological conditions, especially in coma from various causes, appears quite distinctly'. He observed the phenomenon initially only in pathological cases, which he did not describe in detail. Later he recognized the reflex in normal os subjects. Incidentally, the concept that so-called $\vec{O}$ abnormal reflexes are gross exaggerations of the $\overrightarrow{\vec{\omega}}$ normal response under pathological conditions can $\vec{\omega}$ be found in analyses of numerous dysfunctions of the nervous system.

Trömner, unaware of von Sölder's observations $\omega$ 'rediscovered' the reflex in 1918. He was of the $\stackrel{+}{\omega}$ opinion that it was seen only in pathological cases i but did not describe the clinical condition of h\$ patients in detail. He enumerated the clinic diagnoses in 12 patients in whom he observed the reflex (Trömner, 1918, 1922). These included 'righ $\subseteq$ thrombotic apoplexy', 'thrombotic softening wi left-sided paralysis', 'arachnitis cerebri circun $\overrightarrow{0}$ scripta', 'tuberculoma of the pons', 'tumour of the left parietal lobe', 'left temporal lobe glioma', 'left haemorrhagic apoplexy', 'left motor and sensory apoplexy', three cases of amyotrophic lateral sclerosis, and one of Friedreich's ataxia. The o majority of these patients had bilateral corneo- $\frac{\varrho}{\not}$ mandibular reflexes. Trömner felt that it arose from $\stackrel{\mathbb{D}}{\perp}$ suprabulbar lesions of the brain.

The discussion of the subject in the German litera- 3 ture was concerned with the physiological basis for the reflex; whether it was a true reflex or an 'asso-? ciated movement' (von Sölder, 1902, 1904, 1918; Kaplan, 1903; Trömner, 1918, 1922). The discussion was mainly theoretical, since experimental studies:were not done to support the contentions of the various authors.

In a later Russian contribution Goldin described the phenomenon in four out of 100 normal subjects 0 (Goldin, 1936). Ornsteen described a patient with what seemed to be acute polyneuritis of the Guillain-o Barré type in whom numerous synkineses and abnormal associated movements were observed $\sigma$ (Ornsteen, 1935). Among these was an 'upward and N forward' protrusion of the jaw when the eyes blinked. Unfortunately, the effect of unilateral 
blinking or of elicitation of the corneal reflex was not described. In addition, this patient demonstrated lateral deviation of the jaw upon ipsilateral deviation of the eyes. Although cited in the literature as an instance of a corneomandibular reflex in 'polyneuritis', the phenomenon described by Ornsteen seems to be of a different variety.

The phenomenon of contralateral deviation of the jaw with voluntary forced unilateral eyelid closure was reported by Wartenberg (1948). He stated that this phenomenon may be obtained in patients in whom the conventional corneomandibular reflex is elicited. Wartenberg felt that an unilateral corneomandibular reflex signified damage to the supranuclear trigeminal pathways and occurred when the cornea on the side of a hemiplegia was stimulated. According to his hypothesis, the bilateral response was seen in pontine lesions, especially in amyotrophic lateral sclerosis which has affected the suprapontine pathways. Unfortunately, his extensive discussion of this reflex was not documented by clinical descriptions of his patients' neurological status. Wartenberg also noted the occurrence of the corneomandibular reflex in normal subjects, and found that it was quite weak and hard to elicit.

Paulson and Gottlieb (1968) found corneomandibular reflexes in six of 85 patients with senile or presenile dementias. In several of these patients, according to their observations, the phenomenon was more of a mentalis jerk than a movement of the jaw. One wonders if in these patients, the authors were eliciting a 'corneomental' rather than a corneomandibular reflex.

Guiot (1946) stated that the corneomandibular reflex was not seen in healthy subjects, in senility, or in sleep. He felt that it occurred in patients with brain-stem involvement if the state of consciousness improved. Thus, he related the phenomenon to the level and severity of the lesion and to the state of consciousness. He saw the corneomandibular reflex most frequently in acute head injuries, and he also mentioned, but did not describe, a comatose patient with a tumour of the pineal region in whom he observed this phenomenon.

Ansink (1962) performed detailed studies on the incidence and nature of the corneomandibular reflex. His report is marred by the lack of clinical data of his patients and by his failure to state the number of patients studied. Despite this he quoted percentages and stated that he found the reflex in $2 \%$ of patients below 6 months of age, in $6 \%$ of patients between the ages of 20 and 45 , and in $34 \%$ of neurologically normal subjects between the ages of 65 and 92. His method of eliciting the reflex was not the usual method of corneal stimulation. Instead, he forcibly held the eye open and applied a strong stimulus of long duration to the cornea, measurning jaw deviation by a mechanical device. He distinguished between the 'normal' and the 'pathological' corneomandibular reflex without clearly stating his criteria for this differentiation. According to him the pathological reflex is considered so by virtue of its long latent period, greater excursion, and ease of elicitation, while the 'normal reflex' is of short latency, short excursion, and not constantly elicited. Furthermore, Ansink did not state how his measuring device differentiated movements of the mouth or chin (such as the 'corneomental reflex') from jaw deviation per se. On the basis of his observations, which also included necropsy material, he concluded that the corneomandibular reflex signified injury to the brain-stem, and, if present in a supratentorial process, was one of the earliest and most sensitive signs of threatening tentorial herniation. Critical evaluation of his conclusions is difficult to make because of the lack of clinical descriptions in his report.

Most observers of this reflex, including the authors of the present communication, agree on certain phenomenological aspects of the corneomandibular reflex. It is not common under the usual conditions of clinical examination. It may not be elicited unless a relatively firm stimulus is applied to the cornea; a light touch with a wisp of cotton is usually insufficient. It is almost invariably associated with the normal corneal reflex, that is a bilateral blink. There is a general tendency for the reflex to be easier to elicit and to last longer (over two seconds in some cases) the more severe the neurological involvement. In the few normal subjects, or subjects without demonstrable brain disease, in whom this phenomenon is seen it is frequently difficult to elicit, not prominent when elicited and inconstant. Despite the difficulty of elicitation in normal subjects, the corneomandibular reflex is probably an exaggeration of that which occurs normally.

On the basis of the observations reported in the present communication and in the literature a prominent corneomandibular reflex is most commonly seen in patients with severe bilateral cerebral or brain-stem disease in whom there is alteration in the state of consciousness. It does not appear to be of localizing value in indicating the level of the lesion, although in most pathological material in which the phenomenon was observed clinically lesions at or above the pontine level were seen. The phenomenon is also seen in a certain number of patients with neurological diseases in whom the state of consciousness is normal, most prominently in amyotrophic lateral sclerosis, and in a small number of normal subjects. In this latter group the reflex is usually not prominent. 


\section{REFERENCES}

Ansink, J. J. (1962). Physiologic and clinical investigation into 4 brain stem reflexes. Neurology (Minneap.), 12, 320328.

Goldin, A. M. (1936). Corneo-mandibular reflex. Sov. Psychoneur., 12, 99-100.

Guiot, G. (1946). Le réflexe corneo-ptérygoïdien. Le phénomène de la diduction lente du maillaire. Rev. neurol., 78, 48-49.

Kaplan, J. (1903). Zur Frage des 'Corneo-mandibularreflexes'. Neurol. Z Zbl., 22, 910-912.

Ornsteen, A. M. (1935). Palpebromandibular synkinesis in a patient with acute polyneuritis and facial diplegia. Arch. Neurol. Psychiat. (Chic.), 34, 625-630.
Paulson, G., and Gottlieb, G. (1968). Development reflexes: The reappearance of foetal and neonatal reflexes in aged patients. Brain, 91, 37-52.

Trömner, E. (1918). Einen neuen Bulbärreflex (PterygoCornealreflex). Neurol. $\mathrm{Zbl}$., 37, 334.

Trömner, E. (1922). Der Pterygo-Cornealreflex. Z. ges. Neurol. Psychiat., 78, 306-309.

Von Sölder, F. (1902). Der Corneo-mandibularreflex. Neurol. Zbl., 21, 111-113.

Von Sölder, F. (1904). Ueber den Corneo-mandibularreflex. Entegegnung auf J. Kaplan's Einwendungen. Neurol. Zbl., 23, 13-15.

Von Sölder, F. (1918). Bemerkung zu Trömners 'PterygoCorneolreflex'. Neurol. Zbl., 37, 432.

Wartenberg, R. (1948). Winking-jaw phenomenon. Arch. Neurol. Psychiat. (Chic.), 59, 734-753. 